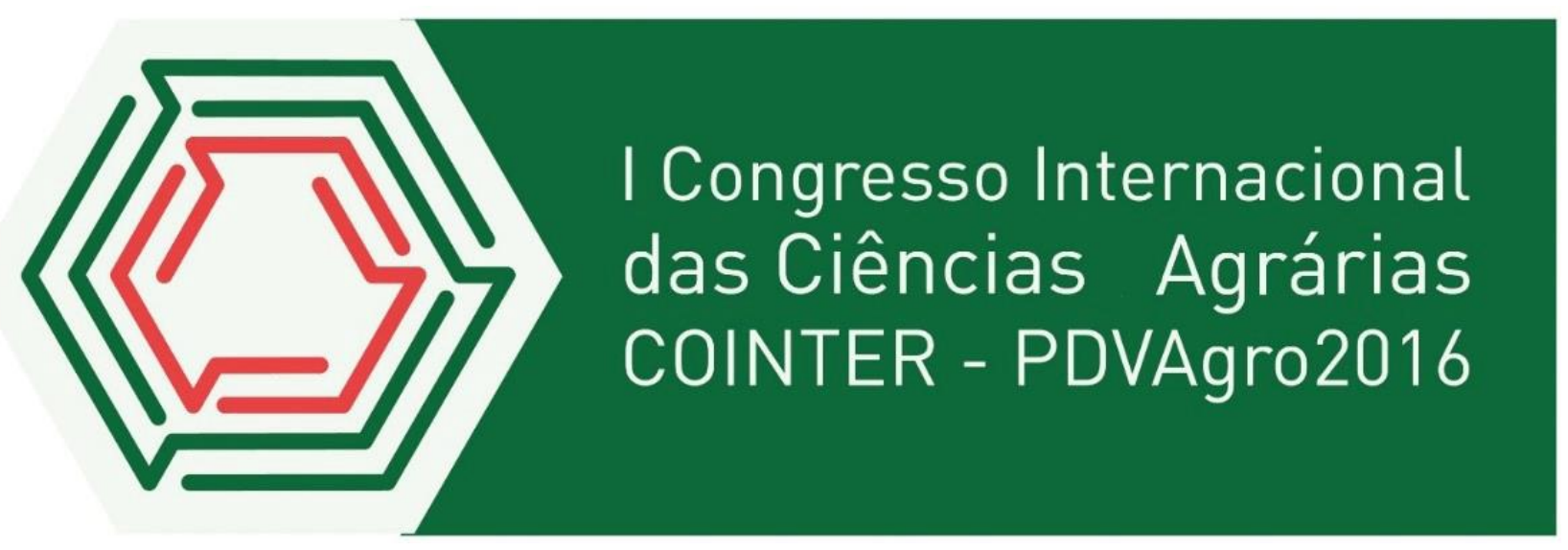

\title{
PRODUÇÃO DE BIOMASSA DE CANA-DE-AÇÚCAR (SCCHARUM SPP.) CULTIVADA SOB DIFERENTES NÍVEIS DE AMÔNIO E NITRATO
}

\author{
Apresentação: Pôster
} Victor Hugo de Farias Guedes ${ }^{1}$; Diego Moura de Andrade Oliveira ${ }^{2}$ José de Arruda Barbosa $^{3}$;
Fernando José Freire ${ }^{4}$; Renato Lemos dos Santos

\section{Introdução}

A cana-de-açúcar (Sccharum spp.) é umas das principais culturas brasileiras. Com perspectiva de aumento de produção na safra 2016/17 de 2,90\% em relação à safra passada. Em números absolutos estima-se uma produção de 684,77 milhões de toneladas de cana-de-açúcar, ante os 665,590 milhões de toneladas na safra 2015/16. A produção de açúcar é estimada na casa dos 39.962,80 mil toneladas, já o etanol produzido nessa mesma safra tende a crescer e chegar ao montante de 27.870.105,60 mil litros (CONAB, 2016).

Esse crescimento de produção pode ser atrelado diretamente à melhoria do manejo da cultura, principalmente a adubação. O nitrogênio (N) é um nutriente muito absorvido pela cana-deaçúcar, sendo superado apenas pelo potássio (K), extraindo 94 a $260 \mathrm{~kg} \mathrm{ha}^{-1}$ de $\mathrm{N}$, variando de acordo com o genótipo (OLIVEIRA et al., 2010).

$\mathrm{Na}$ cana-de-açúcar, a absorção do $\mathrm{N}$ é preferencialmente nas formas amoniacal $\left(\mathrm{NH}_{4}^{+}\right)$e nítrica $\left(\mathrm{NO}_{3}^{-}\right)$, entretanto, em solos aerados a forma nítrica é predominante (ARMAS et al., 1992;

\footnotetext{
${ }^{1}$ Graduando do Curso Bacharelado em Agronomia do Instituto Federal de Pernambuco, Campus Vitória de Santo Antão,victorhguedes1@gmail.com

${ }^{2}$ Graduando do Curso Bacharelado em Agronomia do Instituto Federal de Pernambuco, Campus Vitória de Santo Antão,diegomouradeandradeoliveira@gmail.com

${ }^{3}$ Graduando do Curso Bacharelado em Agronomia do Instituto Federal de Pernambuco, Campus Vitória de Santo Antão, josearruda777@gmail.com
} 
ROBINSON et al., 2011), com $\mathrm{NH}_{4}{ }^{+}$apresentando concentrações até 1000 vezes menor que o NO3(MARSCHNER, 1995). Todavia, para que o $\mathrm{NO}_{3}{ }^{-}$seja absorvido precisa ser reduzido a $\mathrm{NH}_{4}{ }^{+}$nos plastídios da raiz ou nos cloroplastos foliares sendo assim assimilado e utilizado na formação de aminoácidos e proteínas. A enzima redutase do nitrato é fundamental neste processo, por ser responsável pela conversão de $\mathrm{NO}_{3}{ }^{-}$a $\mathrm{NO}_{2}{ }^{-}$(HIREL et al., 2011).

Desta forma, objetivou-se avaliar a produção de biomassa de cana-de-açúcar em diferentes variedades de cana-de-açúcar cultivadas sob diferentes proporções de amônio/nitrato em relação ao $\mathrm{N}$ total.

\section{Fundamentação Teórica}

A importância econômica da cana-de-açúcar está na geração de empregos e renda no meio rural. Isso por ser a matéria-prima para as agroindústrias do açúcar, do álcool e da aguardente (SILVA et al., 2010). Destacando-se, entre as gramíneas tropicais, como a planta de maior potencial para produção de matéria seca e energia por unidade de área, mesmo geralmente tendo apenas um único corte por ano (BOIN, 1985).

$\mathrm{Na}$ assimilação do nitrato $\left(\mathrm{NO}_{3}{ }^{-}\right)$, o nitrogênio do $\mathrm{NO}_{3}{ }^{-}$é convertido em uma forma mais energética, o nitrito $\left(\mathrm{NO}_{2}^{-}\right)$e, então, em uma forma ainda mais energética, o amônio $\left(\mathrm{NH}_{4}^{+}\right)$, e finalmente em nitrogênio-amida da glutamina. Este processo consome o equivalente a 12 ATPs para cada nitrogênio (BLOOM; COLS., 1992). A disponibilidade de $\mathrm{N}$ nas formas $\mathrm{N}_{-} \mathrm{NH}_{4}{ }^{+}$e $\mathrm{N}_{-} \mathrm{NO}_{3}{ }^{-}$, em proporções variáveis no início do desenvolvimento das plantas, pode afetar a emissão e o desenvolvimento de afilhos interferindo sobre o potencial produtivo de culturas como trigo e arroz. Em trigo, o suprimento somente do N-NH4+ reduziu a emissão e o desenvolvimento de afilhos (WANGE; BELOW, 1996).

\section{Metodologia}

Para avaliar o efeito de diferentes proporções de amônio: nitrato no crescimento e na produção de biomassa e indicar a preferência pelas principais formas de $\mathrm{N}$ absorvido em variedades de cana-de-açúcar, realizou-se um experimento em casa de vegetação, no Instituto Federal de Educação Ciência e Tecnologia de Pernambuco - Campus Vitória de Santo Antão (IFPE-CVSA) no período de abril a julho de 2016. Foram utilizadas as variedades RB867515 e RB92579, respectivamente, a mais plantada no país e no nordeste (CHAPOLA et al., 2012).

As mudas foram colocadas em vasos de material plástico, contendo 6 L de solução nutritiva com aeração constante, em sistema hidropônico tipo floating. As variedades foram submetidas a 
cinco soluções com diferentes proporções de amônio /nitrato $\left(\mathrm{NH}_{4}{ }^{+} / \mathrm{NO}_{3}{ }^{-}\right)$: 100:0; 75:25; 50:50; 25:75; 0:100, com base na solução nutritiva de macro e micronutrientes sugerido por Hoagland e Arnon (1950). Desse modo, os tratamentos deste trabalho apresentaram o arranjo fatorial (2 x 5), com quatro repetições por tratamento, totalizando 40 unidades experimentais, distribuídas casualmente em blocos.

Aos 10, 20, 30, 40, 50, 60 dias após o transplantio (DAT) foi realizada a avaliação de contagem de número de folhas verdes e quantidade de perfilho. E ao final do experimento foi realizada a avaliação de massa seca de planta, separando-se a parte aérea da planta do sistema radicular e posteriormente levada à estufa de circulação forçada a $65^{\circ} \mathrm{C}$ até a obtenção de peso constante.

Os dados das variedades de cana-de-açúcar foram submetidos à análise de variância em função das variedades e das proporções de $\mathrm{NH}_{4}{ }^{+} / \mathrm{NO}_{3}{ }^{-}$. Sendo observados efeitos significativos (Teste F, p < 0,05), realizou-se análise de regressão, selecionando-se o modelo que melhor se adapta ao fenômeno, considerando o coeficiente de determinação e a significância dos parâmetros (Teste $\mathrm{t}, \mathrm{p}<0,10)$.

\section{Resultados e Discussões}

Os dados de perfilhamento não apresentaram significância estatística para a variedade RB867515 (Tabela 1) aos 10, 20 e 30 DAT. Já aos 40 DAT observou-se que a dose 0:100 de amônio/nitrato diferenciou estatisticamente da dose 100:0.

Tabela 1. Quantidade de perfilhamento por planta de variedades de cana-de-açúcar submetidas a doses de $\mathrm{NH}_{4}{ }^{+} / \mathrm{NO}_{3}{ }^{-}$ em relação aos dias após o transplantio (DAT).

\begin{tabular}{cccccccccccccc}
\hline \multicolumn{10}{c}{ RB 867515 } \\
\hline $\begin{array}{c}\text { Doses } \\
\left(\mathrm{NH}_{4}{ }^{+} / \mathrm{NO}_{3}{ }^{-}\right)\end{array}$ & 10 & 20 & 30 & 40 & 50 & 60 & 10 & 20 & 30 & 40 & 50 & 60 \\
\hline $0 / 100$ & $8.50 \mathrm{a}$ & $7.75 \mathrm{a}$ & $8.50 \mathrm{a}$ & $7.25 \mathrm{a}$ & $8.50 \mathrm{a}$ & $6.50 \mathrm{a}$ & $8.0 \mathrm{a}$ & $8.75 \mathrm{a}$ & $10.0 \mathrm{a}$ & $5.25 \mathrm{ab}$ & $8.0 \mathrm{a}$ & $8.75 \mathrm{a}$ \\
$25 / 75$ & $7.25 \mathrm{a}$ & $7.25 \mathrm{a}$ & $7.0 \mathrm{ab}$ & $6.50 \mathrm{ab}$ & $5.50 \mathrm{ab}$ & $5.25 \mathrm{a}$ & $7.0 \mathrm{a}$ & $8.25 \mathrm{a}$ & $8.50 \mathrm{ab}$ & $6.0 \mathrm{a}$ & $7.50 \mathrm{a}$ & $6.25 \mathrm{a}$ \\
$50 / 50$ & $8.50 \mathrm{a}$ & $8.25 \mathrm{a}$ & $7.50 \mathrm{ab}$ & $4.50 \mathrm{bc}$ & $4.50 \mathrm{~b}$ & $6.25 \mathrm{a}$ & $7.50 \mathrm{a}$ & $8.0 \mathrm{a}$ & $6.50 \mathrm{bc}$ & $5.25 \mathrm{ab}$ & $5.75 \mathrm{a}$ & $6.75 \mathrm{a}$ \\
$75 / 25$ & $8.25 \mathrm{a}$ & $7.25 \mathrm{a}$ & $5.75 \mathrm{~b}$ & $3.50 \mathrm{c}$ & $4.0 \mathrm{~b}$ & $5.0 \mathrm{a}$ & $7.50 \mathrm{a}$ & $8.0 \mathrm{a}$ & $6.50 \mathrm{bc}$ & $4.75 \mathrm{ab}$ & $5.0 \mathrm{a}$ & $6.75 \mathrm{a}$ \\
$100 / 0$ & $7.0 \mathrm{a}$ & $7.75 \mathrm{a}$ & $6.25 \mathrm{ab}$ & $3.50 \mathrm{c}$ & $3.25 \mathrm{~b}$ & $6.0 \mathrm{a}$ & $7.50 \mathrm{a}$ & $7.0 \mathrm{a}$ & 4.75 & $\mathrm{c}$ & $1.75 \mathrm{~b}$ & $1.0 \mathrm{~b}$ & $1.50 \mathrm{~b}$ \\
\hline $\mathrm{CV}(\%)$ & 22.53 & 18.41 & 16.29 & 22.14 & 27.35 & 12.98 & 11.42 & 10.94 & 14.13 & 40.86 & 32.04 & 31.62 \\
\hline
\end{tabular}

As médias seguidas pela mesma letra na coluna não diferem estatisticamente entre si pelo teste de Tukey (p <0,01).

Ainda para a variedade RB867515 aos 60 DAT verificou-se que as doses 0:100 e 25:75 que apresentavam menores doses de amônio e maiores de nitrato, não diferenciaram estatisticamente entre si, proporcionando maior número de perfilhos por planta de cana-de-açúcar. Resultado 
semelhando ao obtido por Polleto et al. (2010) que observou em experimento com arroz que quando foi aumentada a proporção de $\mathrm{N}-\mathrm{NH}_{4}{ }^{+}$, houve baixa frequência de emissão de ramificações.

Já para a variedade RB92579 (Tabela 1) até os 20 DAT não foi observada nenhuma ramificação de perfilho nós tratamentos 25/75, 75/25, 100/0 amônio/nitrato, apenas para as doses 0:100 e 50:50, houve perfilhamento para essa variedade. A intensificação do perfilhamento apresenta variação entre diferentes cultivares, podendo ocorrer até quatro meses após o plantio, os decréscimo no número de brotações, acontecem em virtude da competição natural por fatores que proporcionam o desenvolvimento da planta, principalmente relacionados a nutrição (CASTRO; CHRISTOFOLETTI, 2005).

Para a variável número de folhas verdes (NFV), observou-se na variedade RB867515 aos 10,20 e 60 DAT, que não houve diferença estatística entre os tratamentos. Entretanto, aos 30 DAT verificou-se que as doses 100:0 e 75:25 amônio:nitrato diferenciaram estatisticamente entre si. Isso provavelmente aconteceu, pois a alta dose de amônio no tratamento 100/0, pode ter provocado menor desenvolvimento das plantas, devido à baixa formação de proteínas e outros compostos nitrogenados que controlam o crescimento, podendo assim, tais plantas, apresentar sintomas que podem ser confundidos com uma deficiência de nitrogênio.

Na variedade RB92579 a dose 0:100 amônio:nitrato, apresentou maior NFV ao longo dos 60 DAT, exceto aos 40 DAT. Constatou-se também um decréscimo no incremento do NFV a medida que as doses de $\mathrm{NH}_{4}^{+}$foram mais maiores. Indicando assim, que os tratamentos com maiores doses de nitrato favoreceram o aumento do NFV no experimento.

Tabela 2. Número de folhas verdes por planta de variedades de Cana-de-açúcar, submetidas a doses de NH4+/NO3-em relação aos dias após o transplantio (DAT).

\begin{tabular}{|c|c|c|c|c|c|c|c|c|c|c|c|c|}
\hline \multirow{3}{*}{$\begin{array}{c}\text { Doses } \\
\text { (NH4+/NO3-) }\end{array}$} & \multicolumn{6}{|c|}{ RB 867515} & \multicolumn{6}{|c|}{ RB 92579} \\
\hline & \multicolumn{6}{|c|}{ DAP } & \multicolumn{6}{|c|}{ DAP } \\
\hline & 10 & 20 & 30 & 40 & 50 & 60 & 10 & 20 & 30 & 40 & 50 & 60 \\
\hline $0 / 100$ & $8.50 \mathrm{a}$ & $7.75 \mathrm{a}$ & $8.50 \mathrm{a}$ & $7.25 \mathrm{a}$ & $8.50 \mathrm{a}$ & $6.50 \mathrm{a}$ & $8.0 \mathrm{a}$ & $8.75 \mathrm{a}$ & $10.0 \mathrm{a}$ & $5.25 \mathrm{ab}$ & $8.0 \mathrm{a}$ & $8.75 \mathrm{a}$ \\
\hline $25 / 75$ & $7.25 \mathrm{a}$ & $7.25 \mathrm{a}$ & $7.0 \mathrm{ab}$ & $6.50 \mathrm{ab}$ & $5.50 \mathrm{ab}$ & $5.25 \mathrm{a}$ & $7.0 \mathrm{a}$ & $8.25 \mathrm{a}$ & $8.50 \mathrm{ab}$ & $6.0 \mathrm{a}$ & $7.50 \mathrm{a}$ & $6.25 \mathrm{a}$ \\
\hline $50 / 50$ & $8.50 \mathrm{a}$ & $8.25 \mathrm{a}$ & $7.50 \mathrm{ab}$ & $4.50 \mathrm{bc}$ & $4.50 \mathrm{~b}$ & $6.25 \mathrm{a}$ & $7.50 \mathrm{a}$ & $8.0 \mathrm{a}$ & $6.50 \mathrm{bc}$ & $5.25 \mathrm{ab}$ & $5.75 \mathrm{a}$ & $6.75 \mathrm{a}$ \\
\hline $75 / 25$ & $8.25 \mathrm{a}$ & $7.25 \mathrm{a}$ & $5.75 \mathrm{~b}$ & $3.50 \mathrm{c}$ & $4.0 \mathrm{~b}$ & $5.0 \mathrm{a}$ & $7.50 \mathrm{a}$ & $8.0 \mathrm{a}$ & $6.50 \mathrm{bc}$ & $4.75 \mathrm{ab}$ & $5.0 \mathrm{a}$ & $6.75 \mathrm{a}$ \\
\hline $100 / 0$ & $7.0 \mathrm{a}$ & $7.75 \mathrm{a}$ & $6.25 \mathrm{ab}$ & $3.50 \mathrm{c}$ & $3.25 \mathrm{~b}$ & $6.0 \mathrm{a}$ & $7.50 \mathrm{a}$ & $7.0 \mathrm{a}$ & $4.75 \mathrm{c}$ & $1.75 \mathrm{~b}$ & $1.0 \mathrm{~b}$ & $1.50 \mathrm{~b}$ \\
\hline $\mathrm{CV}(\%)$ & 22.53 & 18.41 & 16.29 & 22.14 & 27.35 & 12.98 & 11.42 & 10.94 & 14.13 & 40.86 & 32.04 & 31.62 \\
\hline
\end{tabular}

Para massa seca de raiz (MSR), os dados das variedades RB92579 e RB867515 enquadraram-se ao modelo linear de regressão (Figura 1). A variedade RB92579 apresentou maior incremento de MSR até a dose $80 \mathrm{mg} / \mathrm{L}^{-1}$, decaindo nas doses 90 e $100 \mathrm{mg} / \mathrm{L}^{-1}$ de $\mathrm{NH}_{4}{ }^{+}$. Fornecendo 
indícios que a variedade RB867515 suporta doses maiores do íon amônio. Porém ambas as variedades, quando submetidas a doses crescentes de $\mathrm{NH}_{4}{ }^{+}$, apresentaram menores valores de MSR. Alguns autores relatam que o $\mathrm{NH}_{4}{ }^{+}$é tóxico para as células vegetais por atuar como desacoplador entre o fluxo de elétrons e a fosforilação oxidativa.

Figura 1. Massa seca de raiz das variedades RB867515 e RB92579 em relação a diferentes níveis de amônio.

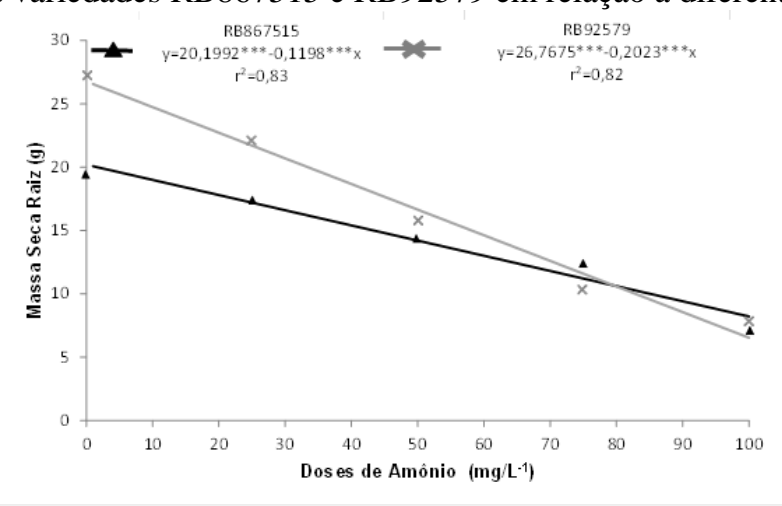

\section{Conclusões}

O desenvolvimento de perfilhos, número de folhas e massa seca de raiz foram modulados pelas proporções entre as formas $\mathrm{N}_{-} \mathrm{NH}_{4}{ }^{+}$e N-NO${ }_{3}{ }^{-}$.

Os tratamentos que possuíam níveis de nitrato mais elevados proporcionaram maior aporte massa seca de raiz, indicando assim, que as variedades RB867515 e RB92579 possuem preferência de absorção do nitrogênio na forma nítrica.

\section{Referências}

ARMAS, R. DE. Influence of Ammonium and Nitrate on the Growth and Photosynthesis of Sugarcane. Journal of Plant Physiology, 140:531-535, 1992.

AZEVEDO, H. M. de. Resposta da cana-de-açúcar a níveis de irrigação e de adubação de cobertura nos tabuleiros da Paraíba. Campina Grande, 2002. 112p. Tese Doutorado. UFCG 2002

BOIN, C.; ALLEONI, G.F.; BIONDI, P. Comparação entre silagem de milho e cana-de-açúcar na alimentação de ruminantes. 2. Efeito da suplementação com uréia na produção de leite. In: REUNIÂO ANUAL DA SOCIEDADE BRASILEIRA DE ZOOTECNIA, 20., 1983, Pelotas. Anais da REUNIÃO ANUAL DA SOCIEDADE BRASILEIRA DE ZOOTECNIA Pelotas: Sociedade Brasileira de Zootecnia, 1983.

CHAPOLA, R. G. Censo varietal 2012. Araras:CCA-UFSCar, 55p. 2012.

COMPANHIA NACIONAL DE ABASTECIMENTO - CONAB. Acompanhamento da safra brasileira de cana-de-açúcar. v. 3 - Safra 2016/17, n. 2 - Segundo levantamento, Brasília, p. 1-72, agosto 2016. 
GILBERT, R.A.; SHINE JUNIOR, J.M.; MILLER, J.D.; RICE, R.W.; RAINBOLT, C.R. The effect of genotype, environment and time of harvest on sugarcane yields in Florida, USA. Field Crops Research, v.95, p.156-170, 2006.

HOAGLAND, D. R., \&amp; ARNON, D. I., The water-culture method for growing plants without soil. Circular. California Agricultural Experiment Station, 2 ed, p. 347, 1950.

WANG, X.T.; BELOW, F.E. Cytokinins in enhanced growth and tillering of wheat induced by mixed nitrogen source. Crop Science, v.36, p.121-126, 1996. 